IJMMS 32:2 (2002) 73-80

PII. S016117120211043X

http://ijmms.hindawi.com

(c) Hindawi Publishing Corp.

\title{
THE GENERALIZED TURNER-BRADLEY-KIRK-PRUITT EQUATION
}

\section{RAY REDHEFFER}

\author{
Received 26 October 2001
}

\begin{abstract}
Several recent results pertaining to nonlinear equations of ecology are applied to a generalization of the Turner-Bradley-Kirk-Pruitt (TBKP) equation, which illustrates a variety of interesting possibilities as regards persistence and extinction. The chief novelty consists in exploiting the value set of the equation, that is, the set of values taken on by the solution as $t$ increases from 0 to $\infty$. This aspect of the subject depends on a new formulation of a condition that was first introduced by Vance and Coddington in 1989.
\end{abstract}

2000 Mathematics Subject Classification: 34A34, 92B05.

1. Introduction. This paper is mainly concerned with a special case of the Kolmogorov equation

$$
\dot{x}=x f(t, x), \quad x(0)=x_{0}>0,
$$

where $f(t, x)$ is continuous at least for $t \geq 0$ and $x>0$. As a matter of convention, conditions involving lim, limsup, or liminf pertain to behavior as $t \rightarrow \infty$. A hypothesis involving $t$ without further explanation, such as $d(t)<k e(t)$, is understood to hold for all $t \geq 0$.

An interesting example of a Kolmogorov equation, introduced by Turner et al. [3], is

$$
\dot{x}=c x^{1-n p}\left(k^{n}-x^{n}\right)^{1+p} .
$$

The parameters $p, n, k, c$ are constants with $n, k, c$ positive and $p>-1$. As seen in [3], (1.2) includes a number of special cases that have long played a role in ecology.

In [2] it was observed that the expression $\left(k^{n}-x^{n}\right)^{1+p}$ is usually meaningless when $x>k$, and this expression was therefore replaced by the odd power function

$$
\left(k^{n}-x^{n}\right)^{\{1+p\}}=\operatorname{sgn}\left(k^{n}-x^{n}\right)\left|k^{n}-x^{n}\right|^{1+p} .
$$

Next, $c$ was replaced by a positive continuous function $c(t)$, and an additional term of the form $d(t) x-e(t) x^{2}$ was introduced, where $d(t)$ and $e(t)$ are continuous with $e(t) \geq 0$ but $d(t)$ unrestricted as to sign. The equation so obtained is

$$
\dot{x}=c(t) x^{1-n p}\left(k^{n}-x^{n}\right)^{\{1+p\}}+d(t) x-e(t) x^{2}, \quad x(0)=x_{0}>0 .
$$

In [2] it is called the generalized TBKP equation because of its genesis from (1.2). This terminology and the above assumptions on $c(t), d(t)$, and $e(t)$ are retained here. Under these assumptions it is not hard to show that all solutions of (1.4) exist for $0 \leq t<\infty$ and are positive. The case where $c$ is constant and $d(t)=e(t)=0$ extends 
the results in [3] to allow $x>k$. But more important is the presence of the arbitrary functions $c(t), d(t)$, and $e(t)$, which leads to a theory of increased scope.

Our main objective is to show how some recently improved results for (1.1) can be applied to (1.4). The improvement consists mainly in the generalized VanceCoddington condition, which leads to Theorems 2.4 and 2.5 below. In their original unimproved forms, these theorems give little or no information about the particular problems of interest here.

2. General results. In each of the following theorems, $x$ denotes a solution of (1.1). As in [1], we introduce the conditions

$$
\begin{aligned}
& 0 \leq s \leq t \Longrightarrow \int_{s}^{t} f(\tau, a) d \tau \geq-A, \\
& 0 \leq s \leq t \Longrightarrow \int_{s}^{t} f(\tau, b) d \tau \leq B,
\end{aligned}
$$

where $a, b, A, B$ are constants. The notations $f(t, \downarrow a)$ and $f(t, a \downarrow)$ mean, respectively

$$
\xi \leq a \Longrightarrow f(t, \xi) \geq f(t, a), \quad \xi \geq a \Longrightarrow f(t, \xi) \leq f(t, a) .
$$

The following theorem from [1] is an improvement of an earlier result of Vance and Coddington [4].

THEOREM 2.1. Suppose that $f(t, \downarrow a), f(t, b \downarrow)$, and that (2.1) holds with constants $A \geq 0, B \geq 0, a>0, b>0$. Then

$$
\min \left(x_{0}, a\right) e^{-A} \leq x(t) \leq \max \left(x_{0}, b\right) e^{B}, \quad 0 \leq t<\infty .
$$

The next result is implicit in [2].

THEOREM 2.2. Suppose there exist arbitrarily small values $b>0$ such that

$$
f(t, b \downarrow), \quad \liminf \int_{0}^{t} f(\tau, b) d \tau=-\infty .
$$

Then the solutions of (1.1) satisfy $\inf x(t)=0$. If $f(t, \xi)$ is continuous at $\xi=0$ and

$$
f(t, 0 \downarrow), \quad 0 \leq s \leq t \Longrightarrow \int_{s}^{t} f(\tau, 0) d \tau \leq B
$$

for some constant $B$, then $\liminf x(t)=0 \Rightarrow \lim x(t)=0$.

Theorem 2.3 gives existence of a threshold below which the population goes to extinction.

THEOREM 2.3. Suppose that there exists a continuous function $\lambda(t) \geq 0$ such that

$$
0<\xi<\delta \Longrightarrow f(t, \xi) \leq-\lambda(t)
$$

where $\int_{0}^{\infty} \lambda(t) d t=\infty$. Then $x(0)<\delta \Rightarrow \lim x(t)=0$. 
Proof. So long as $x(t)<\delta$ we have $\dot{x} \leq 0$, hence $x(0)<\delta \Rightarrow x(t)<\delta$. Equation (2.6) gives $\dot{x} \leq-x(t) \lambda(t)$ and this gives the conclusion.

The following generalization of a hypothesis of Vance and Coddingtion (VC) was introduced in [2].

The GENERALIZED VC Condition. Let $I$ be an open interval, finite or infinite. The function $f(t, x)$ satisfies the generalized VC condition relative to $I$ if there is a continuous function $\lambda \geq 0$, independent of $I$, and a positive constant $\delta$ depending on $I$, such that

$$
\int_{0}^{\infty} \lambda(t) d t=\infty, \quad \xi \in I \Longrightarrow f_{x}(t, \xi) \leq-\delta \lambda(t)
$$

VALUE INTERVAL. A value interval $I(x)$ for the solution $x$ is any open interval containing all values $x(t)$.

In [2] it was seen that these definitions lead to a stability theorem that generalizes [4, Theorem 5].

THEOREM 2.4. Suppose that $x, y$ are two solutions with a common value interval $I=I(x)=I(y)$ relative to which the generalized VC condition holds. Then

$$
\inf y(t)>0 \Longrightarrow \lim \frac{y(t)}{x(t)}=1
$$

The next result has not been stated heretofore, so far as we know, hence is proved in full.

THEOREM 2.5. Given $\epsilon>0$, suppose that $f(t, \xi)$ satisfies the generalized VC condition relative to each interval $(c, 2 c), 0<c<\epsilon$, that $f(t, b \downarrow)$ for $0<b<\epsilon$, and that $f(t, \xi)$ is continuous at $(t, 0)$. If

$$
F(t)=\int_{0}^{t} f(\tau, 0) d \tau, \quad \Lambda(t)=\int_{0}^{t} \lambda(\tau) d \tau
$$

then $\liminf F(t) / \Lambda(t) \leq 0 \Rightarrow \liminf x(t)=0$.

When used together with (2.5), this extends [4, Theorem 4] in three respects: the VC condition is assumed only in a value interval, the hypothesis $f_{x}(t, \xi) \leq 0$ is replaced by generalized monotonicity for small $\xi$, and the condition $\liminf F(t) / \Lambda(t) \leq 0$ is much weaker than the hypothesis $F(t) \leq \beta$ required in [4].

Proof. Choose $b$ with $0<b<\epsilon$ and set $c=b / 2$. Then

$$
f(t, b)-f(t, c)=c f_{x}(t, \xi) \leq-c \delta \lambda(t)
$$

where $\delta$ is a positive constant depending on $c$. The hypothesis implies $f_{x} \leq 0$ for $x<\epsilon$, so $f(t, c) \leq f(t, 0)$. Hence

$$
f(t, b) \leq f(t, 0)-c \delta \lambda(t)
$$


This gives

$$
\int_{0}^{t} f(\tau, b) d \tau \leq F(t)-c \delta \Lambda(t)
$$

and the result follows from the first statement in Theorem 2.2.

3. Examples. In the generalized TBKP equation,

$$
f(t, x)=c(t) x^{-n p}\left(k^{n}-x^{n}\right)^{\{1+p\}}+d(t)-x e(t) .
$$

When $y \neq 0$ the equation $(d / d y) y^{\{\alpha\}}=\alpha|y|^{\alpha-1}$ holds for any constant $\alpha$ and yields

$$
f_{x}(t, x)=-n c(t)\left(x^{n}+p k^{n}\right) x^{-n p-1}\left|x^{n}-k^{n}\right|^{p}-e(t) .
$$

Several results pertaining to (1.4) were given in [2]. Instead of duplicating these we give further examples that are not included there. Most of these involve the notion of value interval introduced in Section 2.

In each of the following examples, $x$ denotes a solution of (1.4).

EXAMPLE 3.1. Suppose that $p<0, d(t)<0$, and

$$
\sup \frac{c(t)}{|d(t)|}<\infty, \quad \int_{0}^{\infty} d(t) d t=-\infty
$$

Then there exists $\delta>0$ such that $x(0)<\delta \Rightarrow \lim x(t)=0$.

This follows from Theorem 2.3, but imposes an artificially strong condition on $d(t)$. Example 3.2 also follows from Theorem 2.3 and has only a weak condition on $d(t)$.

EXAMPLE 3.2. Suppose that $p<-1 / n, d(t) \leq 0$, and

$$
\sup \frac{c(t)}{e(t)}<\infty, \quad \int_{0}^{\infty} e(t) d t=\infty
$$

Then there exists $\delta>0$ such that $x(0)<\delta \Rightarrow \lim x(t)=0$.

The next examples pertain to stability. Information about the interval $I$ of values is given by conditions such as

$$
\sup x(t)<k \quad \text { or } \quad \inf x(t)>k \text {, }
$$

which are realistic only if they can be deduced from the corresponding initial conditions. Further discussion is given in Section 4. It suffices here to say that the equation

$$
\dot{x}(t)=d(t) k-e(t) k^{2}
$$

holds for $x=k$, and shows that the line $x=k$ is a repeller from below or above according as

$$
d(t)-e(t) k<0 \quad \text { or } \quad d(t)-e(t) k>0 .
$$


We then have

$$
x(0)<k \Rightarrow x(t)<k \quad \text { or } \quad x(0)>k \Longrightarrow x(t)>k,
$$

in the two cases respectively. In a like manner, all solutions are bounded away from 0 and $\infty$ if there exist positive constants $\delta, \mu$ such that

$$
\delta e(t) \leq d(t) \leq \mu(c(t)+e(t)) .
$$

The left-hand inequality makes $f(t, x)>0$ when $x<\delta$ and the right-hand inequality makes $f(t, x)<0$ when $x>\mu$, assuming, as we may, that $\delta$ is small and $\mu$ is large. A modification of these ideas gives more subtle conditions introduced later.

EXAMPLE 3.3. Assuming $-1<p<0$, let $x$ and $y$ be two solutions satisfying

$$
x(t) \geq k|p|^{1 / n}, \quad y(t) \geq k|p|^{1 / n} .
$$

Suppose, further, that

$$
\int_{0}^{\infty} e(t) d t=\infty
$$

Then $\lim x(t) / y(t)=1$.

We have $f_{x}(t, x) \leq-e(t)$ for $x$ in the common value interval $I$ of $x$ and $y$ and the result follows from Theorem 2.4.

EXAMPLE 3.4. Suppose that $p>0$ and that

$$
\int_{0}^{\infty}(c(t)+e(t)) d t=\infty .
$$

Let $x(t)$ and $y(t)$ be two solutions satisfying

$$
\sup x(t)<k, \quad \sup y(t)<k, \quad \inf y(t)>0 .
$$

Then $\lim x(t) / y(t)=1$ and hence also $\lim |x(t)-y(t)|=0$.

In this case $f_{x}(t, x) \leq-\delta c(t)-e(t)$ for $x \in I$, where $\delta$ is a positive constant, and the result follows from Theorem 2.4. The same method gives Examples 3.5 and 3.6.

EXAMPLE 3.5. Assuming $-1<p<0$, suppose (3.12) holds. Let $x(t)$ and $y(t)$ be two solutions satisfying $\sup x(t)<k$, sup $y(t)<k$, and

$$
\inf x(t)>k|p|^{1 / n}, \quad \inf y(t)>k|p|^{1 / n} .
$$

Then $\lim x(t) / y(t)=1$ and hence also $\lim |x(t)-y(t)|=0$.

EXAMPLE 3.6. Suppose that (3.12) holds. Let $x(t)$ and $y(t)$ be two solutions satisfying inf $x(t)>k$, inf $y(t)>k, \sup x(t)<\infty$, and sup $y(t)<\infty$. Then $\lim x(t) / y(t)=1$ and hence also $\lim |x(t)-y(t)|=0$. 
4. The set of values. To use the foregoing results effectively, one must know something about the set of values $\{x(t)\}$ without knowing the solution. For the reader's convenience, we recall that in (1.1), $\dot{x}=x f(t, x), x(0)=x_{0}>0$. With $\alpha$ and $\beta$ positive constants, clearly

$$
\begin{aligned}
& x(0)>\alpha, \quad f(t, \alpha)>0 \Longrightarrow x(t)>\alpha, \\
& x(0)<\beta, \quad f(t, \beta)<0 \Longrightarrow x(t)<\beta .
\end{aligned}
$$

Thus $(\alpha, \beta)$ is a value interval for $x(t)$ if for $x(0)$. Since our theorems can be applied for $t \geq T$, where $T$ is arbitrarily large, it suffices to have $x(t) \in I(x)$ only for large $t$. However we sometimes need $\inf x(t)>\alpha, \sup x(t)<\beta$ or both. This is accomplished by consideration of $(\alpha+\epsilon, \beta-\epsilon)$. The computation is simplified by finding $f(t, \alpha)$ and $f(t, \beta)$, and using continuity to estimate the effect of $\epsilon$.

Using these ideas we discuss the function

$$
f(t, x)=N(x) c(t)+d(t)-x e(t), \quad N(x)=x^{-n p}\left(k^{n}-x^{n}\right)^{\{1+p\}}
$$

associated with the generalized TBKP equation $\dot{x}=x f(t, x)$. It is helpful to note that $N(x)$ has the same sign as $k-x$ and that $|k-x|<\epsilon \Rightarrow|N(x)|<\eta$ where $\eta \rightarrow 0$ with $\epsilon$. Furthermore,

$$
N(\delta)=\delta^{-n p}\left(k^{n}-\delta^{n}\right)^{1+p}, \quad N(\mu)=\mu^{-n p}\left(\mu^{n}-k^{n}\right)^{1+p}
$$

for $\delta<k$ and $\mu>k$, respectively.

EXAMPLE 4.1. Since $f(t, k)=d(t)-k e(t)$, the procedure gives

$$
\begin{aligned}
& x(0)<k \Longrightarrow \sup x(t)<k \quad \text { if } \sup \frac{d(t)-k e(t)}{c(t)+e(t)}<0, \\
& x(0)>k \Longrightarrow \inf x(t)>k \quad \text { if } \inf \frac{d(t)-k e(t)}{c(t)+e(t)}>0 .
\end{aligned}
$$

EXAMPLE 4.2. A necessary and sufficient condition for $N^{\prime}(x) \leq 0$ is that $x^{n}+$ $p k^{n} \geq 0$. This holds if $p \geq 0$ or if $-1<p<0$ and $x(t) \geq k|p|^{1 / n}$. By a short calculation

$$
N\left(k|p|^{1 / n}\right)=|p|^{|p|}(1-|p|)^{1-|p|} k^{n} \geq \frac{1}{2} k^{n}
$$

with equality only when $p=1 / 2$. Hence

$$
x_{0}>k|p|^{1 / n} \Longrightarrow x(t)>k|p|^{1 / n} \quad \text { if } \frac{1}{2} k^{n} c(t)+d(t) \geq|p|^{1 / n} k e(t),
$$

provided that there is strict inequality in the latter relation when $p=1 / 2$.

If $f(t, \delta)>0$ for all $t$, with $\delta>0$ but arbitrarily small, then $\inf x(t)>0$. Since $N(\delta)>0$ a sufficient condition is $d(t) \geq \delta e(t)$. But this can be improved by exploiting the term $N(\delta) c(t)$ when $n p \geq-1$, as seen by the following example.

EXAMPLE 4.3. The condition inf $x(t)>0$ follows when $p>0$ from

$$
\inf \frac{d(t)}{c(t)}>-\infty, \quad \sup \frac{e(t)}{c(t)}<\infty
$$


when $p=0$ from

$$
\liminf \frac{d(t)}{c(t)}>-k^{n}, \quad \sup \frac{e(t)}{c(t)}<\infty,
$$

when $-1<n p<0$ from

$$
\liminf \frac{d(t)}{c(t)} \geq 0, \quad \sup \frac{e(t)}{c(t)}<\infty,
$$

and when $n p=-1$ from

$$
\liminf \frac{d(t)}{c(t)} \geq 0, \quad \limsup \frac{e(t)}{c(t)}<k^{n-1} .
$$

The following sharper result also holds.

EXAMPLE 4.4. When $p=0$ the conclusion $\inf x(t)>0$ follows from

$$
\liminf \frac{c(t) k^{n}+d(t)}{c(t)+e(t)}>0 .
$$

5. The case $p=0$. Existence of $f(t, 0)$ requires $p \leq 0$ and the condition $f_{x} \leq 0$ for $x$ near 0 requires $p \geq 0$. Hence $p=0$ is the only case in which both conditions hold. Throughout this section we set $p=0$, so the differential equation is

$$
\dot{x}=c(t) x\left(k^{n}-x^{n}\right)+d(t) x-e(t) x^{2} .
$$

Thus, $f(t, x)=c(t)\left(k^{n}-x^{n}\right)+d(t)-e(t) x$ and

$$
f_{x}(t, x)=-c(t) n x^{n-1}-e(t) .
$$

We use the abbreviations

$$
\lambda(t)=c(t)+e(t), \quad f(t)=c(t) k^{n}+d(t) .
$$

EXAMPLE 5.1. Suppose that the functions

$$
\Lambda(t)=\int_{0}^{t} \lambda(\tau) d \tau, \quad F(t)=\int_{0}^{t} f(\tau) d \tau,
$$

satisfy $\Lambda(\infty)=\infty$ and $\liminf F(t) / \Lambda(t)=0$. Then the solutions of (5.1) satisfy

$$
\inf x(t)=0 \quad \text { if } n \geq 1, \quad \inf x(t)=0 \text { or } \sup x(t)=\infty \quad \text { if } n<1 .
$$

The proof depends on the generalized VC condition, which in turn follows from

$$
f_{x}(t, \xi) \leq-\lambda(t) \gamma(\xi),
$$

where $\gamma(\xi)=\min \left(1, n \xi^{n-1}\right)$. We need $\gamma(\xi)$ only for $\xi$ in a value interval $I$ for $x(t)$. Hence

$$
\begin{aligned}
& n>1, \quad \inf x(t)>0 \Longrightarrow \inf \gamma(\xi)>0, \\
& n<1, \quad \sup x(t)<\infty \Longrightarrow \inf \gamma(\xi)>0 .
\end{aligned}
$$

Example 5.1 now follows from Theorem 2.5. 
The next example implies the opposite conclusion inf $x(t)>0$ and shows that the conditions leading to Example 5.1 are in several respects sharp.

EXAMPLE 5.2. Suppose that there are positive constants $\delta, \mu$ such that

$$
\delta \int_{S}^{t} \lambda(\tau) d \tau \leq \int_{S}^{t} f(\tau) d \tau \leq \mu \int_{S}^{t} \lambda(\tau) d \tau
$$

holds for $0 \leq s \leq t$. Then all solutions of (5.1) are bounded away from 0 and $\infty$.

The conditions (2.1) for (5.1) are equivalent to

$$
\int_{s}^{t}\left(a^{n} c(t)+e(t) a\right) d t-A \leq \int_{s}^{t} f(t) d t \leq \int_{s}^{t}\left(b^{n} c(t)+e(t) b\right) d t+B .
$$

We choose $a>0$ small in the first and $b$ large in the second. Since $c(t)>0$ and $e(t) \geq 0$, the inequalities hold for some $a, b>0$ if and only if (5.8) holds for some positive constants $\delta, \mu$. Example 5.2 follows from Theorem 2.1.

EXAMPLE 5.3. Assuming $\Lambda(\infty)=\infty$, let $x(t)$ and $y(t)$ be two solutions of (5.1) with $\inf y(t)>0$. Then $\lim x(t) / y(t)=1$ if $n>1$ and $\inf x(t)>0$, or if $n=1$, or if $n<1$ and both $x(t)$ and $y(t)$ are bounded.

This follows from (5.6) and Theorem 2.4.

\section{REFERENCES}

[1] R. Redheffer, Generalized monotonicity, integral conditions and partial survival, J. Math. Biol. 40 (2000), no. 4, 295-320.

[2] R. Redheffer and R. R. Vance, A sharpened stability theorem concerning population growth in a variable environment, to appear in Int. J. Math. Math. Sci.

[3] M. E. Turner, E. L. Bradley Jr., K. A. Kirk, and K. M. Pruitt, A theory of growth, Math. Biosci. 29 (1976), 367-373.

[4] R. R. Vance and E. A. Coddington, A nonautonomous model of population growth, J. Math. Biol. 27 (1989), no. 5, 491-506.

RAY REDHEFFER: DEPARTMENT OF MATHEMATICS, UCLA, Los ANGELES, CA 90095-1555, USA 


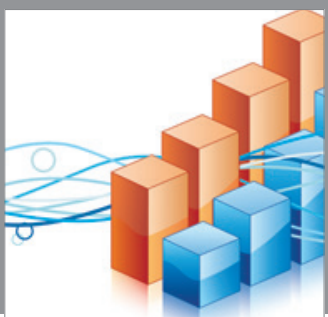

Advances in

Operations Research

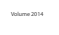

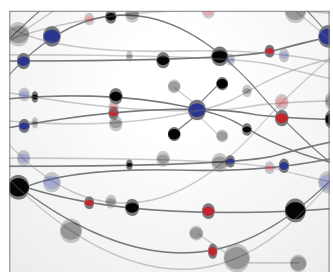

\section{The Scientific} World Journal


International Journal of

Mathematics and

Mathematical

Sciences
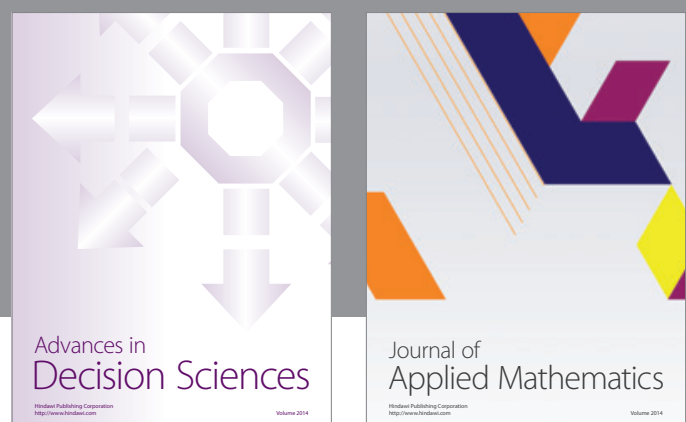

Journal of

Applied Mathematics
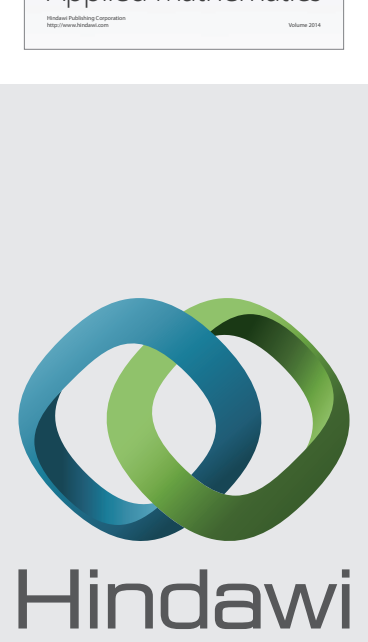

Submit your manuscripts at http://www.hindawi.com
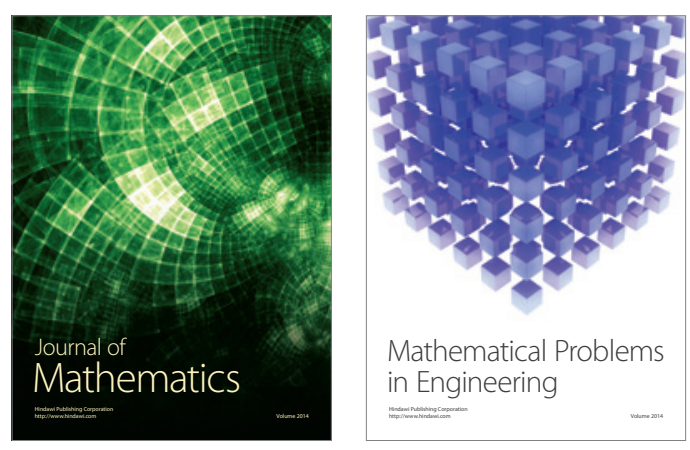

Mathematical Problems in Engineering
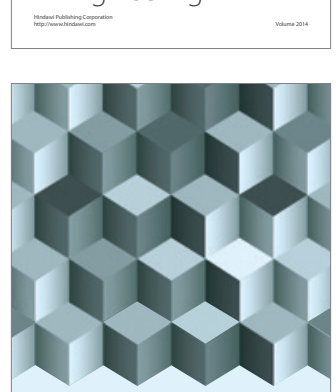

Journal of

Function Spaces
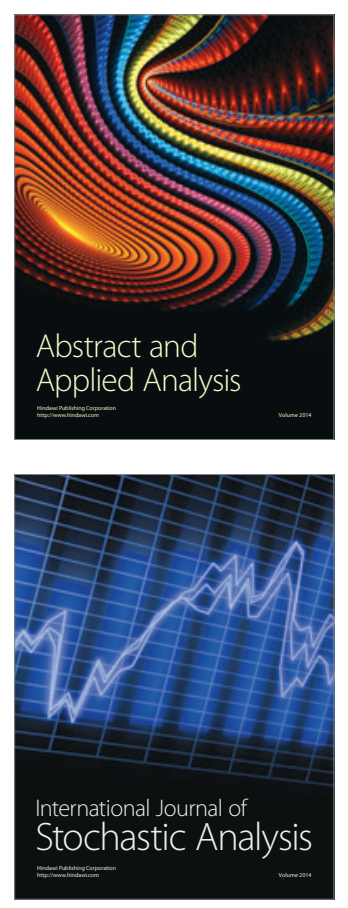

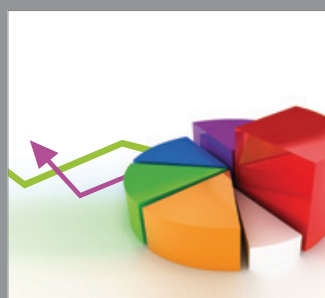

ournal of

Probability and Statistics

Promensencen
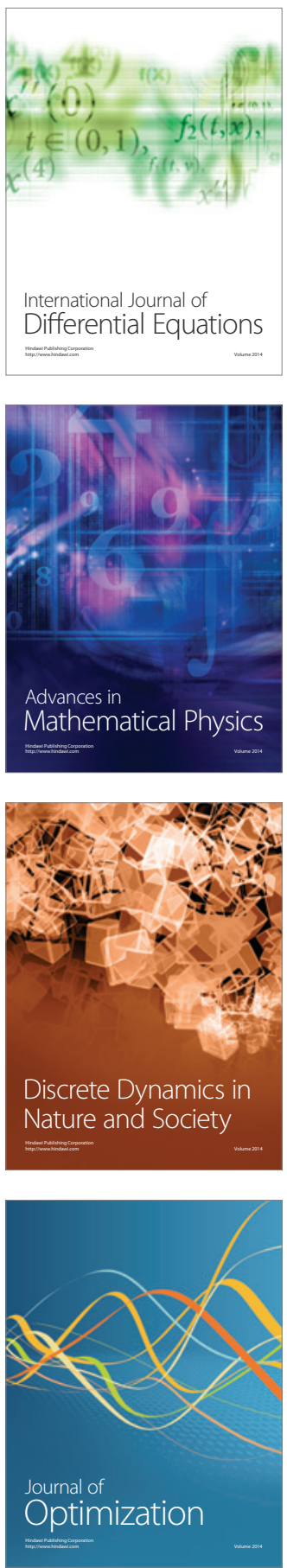